\title{
Platform, Meet APPs: Pricing AND QUAlity Under CROSS-SIDED EXTERNALITIES
}

\author{
Raymond G. Sin \\ Hong Kong University of Science and Technology \\ rsin@ust.hk \\ and \\ Jia Jia \\ Hong Kong University of Science and Technology \\ jiajia@ust.hk
}

\section{BACKGROUND AND MOTIVATION}

Our study is motivated by the observation that two-sided platforms often exercise control on access to and transactions on at least one side of the market. For example, Apple's rigorous approval process and recent policies that prohibit cross-platform app-building tools is an indication of imposing control on both access and quality of its app market. Extant literature on two-sided markets fails to accommodate platform's interventions on participation by either side (i.e. consumers and sellers of complementary products) of the market because it typically focuses on a homogeneous population (c.f. Caillaud and Jullien 2003; Armstrong 2006). Our work aims to bridge this gap and provides the underlying rationale for the platform firm's governance structure and optimal pricing decisions. 
We model a profit-maximizing platform (e.g. iPhone and iPad, Android phones, tablets by

Samsung and LG, game consoles) with cross-side network effects. Our setup consists of two developers who create and sell products (e.g. apps, software, games) in a complementary goods market. Unlike conventional models, we assume participants from both sides of the platform to be heterogeneous. Specifically, consumers are horizontally differentiated in their values for the standalone platform, and incur a "fit" cost of travelling from their ideal locations to purchasing the platform product. Further, developers also differ in their efficiencies in creating quality product in the complementary goods market; this implies that the quality levels of complementary products may also be heterogeneous as well.

By explicitly modeling competition among developers with differential capabilities, we are able to capture an interesting tradeoff confronted by the platform firm: on one hand, accommodating both developers in the complementary product market allows it to fulfill consumers' desire on variety; while on the other, ruling out the less efficient developer ensures higher product quality but decreases consumers' desire to purchase the platform. Our contributions to extant literature are two-fold: First, we identify the conditions under which this quality-variety tradeoff favors one over the other. Second, we explore the effectiveness of a non-price mechanism on the platform firm's profitability and effective intervention on the complementary goods market. 


\section{LITERATURE REVIEW}

There is a rich literature on two-sided markets involving platforms. A platform may include "physical components, tools and rules to facilitate development, a collection of technical standards to support interoperability, or any combination of these things" (Boudreau 2010). Serving as a stable nexus or foundation, a platform also serves as a "technological bottleneck" (Armstrong 2006; Rochet and Tirole 2006). The entity holding property rights over a platform possesses the right to exclude others from the entire system, therefore enjoying the privilege to impose or remove restrictions on use, development, and commercialization of the platform (Boudreau 2010). Platform owners can exploit these privileges to develop tools, such as common ownership of various components suppliers (Bakos and Katsamakas 2008), long-term contracts, and industry-wide standard-setting bodies, so as to achieve coordination among firms that is extensively required by systems competition (Katz and Shapiro 1994).

The need for coordination sometimes goes beyond the supply side and includes end consumers as well (Katz and Shapiro 1994). Besides coordinating consumers to enlarge the network and increase the value of membership for each user, coordination can also involve both sides of the market by facilitating interactions between consumers and producers of complementary components. Such cross-side coordination has significant implications on the willingness to trade on the platform due to the net surpluses resulting from potential interactions of the two sides (Rochet and Tirole 2006). 
Existing research investigates how a platform can employ pricing strategies to accomplish such cross-side coordination, enhancing and extracting surpluses from both sides of the market (Economides and Katsamakas 2006; Lin et al. 2011). Previous studies show that factors influencing the equilibrium platform price include magnitude of the cross-side externalities, formats of fees (e.g., lump-sum or transaction-based), and agents multi-homing considerations if several platforms coexist (Armstrong 2006; Hagiu 2006). The effects of various non-price instruments (e.g., employing screening procedures or raising application development costs for the platform), however, remain largely unaddressed in extant literature.

Another aspect that has not received sufficient attention in the context of two-sided markets is consumer demand for product variety (Hagiu 2009). The widely-adopted assumption of a homogeneous population (Caillaud and Jullien 2003; Armstrong 2006) somewhat contradicts with the fact that a variety of contents are available from independent parties engaging in most two-sided markets in reality. Under this homogeneity assumption, providers of complementary goods are indistinguishable and thus unanimous in their choices; the incentives of these providers in allocating efforts to supply the complementary goods is ignored, resulting in a misled conclusion about intra-platform competition among providers of complementary products. Our work sheds light on how non-price instruments can be employed in combination with revenue-sharing with developers to achieve an optimal outcome. It is also 
one of the first to examine the design issue of a platform firm when forming its intervention strategies for the complementary goods market.

\section{MODEL}

Extant theories on two-sided markets examine externalities (in terms of market size or welfare) generated by one side of the market for another, typically as a function of the number of participants on a given side. It is important to note that, in this work, we do not assume a positive correlation between the presence of participants and network effects; but rather, we investigate the dynamics between a platform firm and developers in a complementary goods market through their strategic considerations, such as quality investment and pricing decisions, and examine the corresponding effects on platform profitability.

There are three players in the model; i.e. a platform firm, third-party developers, and infinite consumers. The platform firm produces and sells a platform product (for example, the iPhone), while independent developers develop and sell complementary products for the platform (for example, apps). Throughout the manuscript, we shall use the terms "complementary goods" and "apps" interchangeably. The platform product is independently valued $\bar{r}$ by heterogeneous consumers that are uniformly distributed along a product space captured by an infinite line as in Salop (1979). This assumption rules out the trivial scenario that the platform firm serves the entire market, and allows us to focus on the cross-side externalities between the platform and apps where attractiveness of the platform product influences the demand for apps, while the provision of apps in the complementary goods market may also 
influence the demand for the platform product. The platform firm incurs a marginal cost $c$ of

producing the platform product and offers it to the consumers at a price $f$. The firm also shares a

predetermined ratio $\alpha \in 0,1 \quad$ of the revenues generated from app sales by the developers in the

complementary goods market ${ }^{1}$.

The utility that a consumer derives from purchasing the platform product is $u_{f}=\bar{r}-x-f$, where $x$ denotes that distance between the consumer's location (i.e. her ideal preference point) from that of the platform product. Without loss of generality, the per unit distance "fit cost" incurred by the consumer is normalized to 1 .

Upon acquiring the platform, consumers then decide whether or not to purchase apps in the complementary goods market. This market is served by two developers who independently decide whether or not to produce app for the platform; and if so, at what price (denoted by $t_{i}$ ) to offer it to the consumers. The two developers differ in their production efficiency, with marginal development costs $\theta_{1}$ and $\theta_{2}>\theta_{1}$ respectively. Each developer produces at most one product with a one-time development cost $W \quad q, \theta=\frac{1}{2} \theta q^{2}$ for an app of quality $q$ with zero marginal cost. A consumer purchases at most one unit of each app, and derives a utility of $u_{a}=\max 0, q_{1}-t_{1}+\max \quad 0, q_{2}-t_{2}$. Hence, a consumer's overall utility can be represented by:

\footnotetext{
${ }^{1}$ We treat $\alpha$ as an exogenous parameter and express the optimal platform price as a function of this revenue-sharing ratio for the following reasons: first, the platform owners need to determine and announce the revenue-sharing rule before app developers commit to developing software for the platform, such a rule is determined before the realization of the qualities and prices of the apps. Second, the revenue-sharing agreement is more sticky, while platform price is in direct control of the platform owner and is more flexible. Revenue-sharing is essentially a contractual agreement between the platform owner and multiple app developers, and it is not easy for the platform to change it from time to time.
} 


$$
U=\bar{r}-x-f+\max \quad 0, q_{1}-t_{1}+\max \quad 0, q_{2}-t_{2}
$$

The marginal consumers who are indifferent between purchasing the platform and opting out of the market is the solution of $x$ to $\bar{r}-f-x+\max 0, q_{1}-t_{1}+\max \quad 0, q_{2}-t_{2}=0$. Therefore, the demand for the platform product can be represented by $\bar{r}-f+\max 0, q_{1}-t_{1}+\max 0, q_{2}-t_{2}$. Figure 1 describes the game sequence.

Figure 1: Game Sequence

\begin{tabular}{|c|c|c|c|c|}
\hline $\begin{array}{c}\text { Stage 1: Platform } \\
\text { Pricing }\end{array}$ & $\begin{array}{l}\text { Stage 2: App } \\
\text { Development }\end{array}$ & $\begin{array}{l}\text { Stage 3: App } \\
\text { Pricing }\end{array}$ & $\begin{array}{l}\text { Stage 4: Platform } \\
\text { Adoption }\end{array}$ & $\begin{array}{l}\text { Stage 5: App } \\
\text { Consumption }\end{array}$ \\
\hline & $\downarrow$ & $\downarrow$ & & \\
\hline $\begin{array}{l}\text { The Platform firm } \\
\text { decides the price for } \\
\text { its product }(f)\end{array}$ & $\begin{array}{l}\text { i. Quality Delegation } \\
\text { App developers decide } \\
\text { qualities for their own } \\
\text { products }\left(q_{1} \text { and } q_{2}\right) \\
\text { ii. Quality Control } \\
\text { Platform firm decides } \\
\text { qualities for the apps } \\
\text { without knowing the exact } \\
\text { development costs of the } \\
\text { developers }\end{array}$ & $\begin{array}{l}\text { Given } q_{1} \text { and } q_{2} \text {, } \\
\text { developers decide } \\
\text { prices for their own } \\
\text { apps }\left(t_{1} \text { and } t_{2}\right) \text {. }\end{array}$ & $\begin{array}{l}\text { Observing } f,\left\{q_{1}, t_{1}\right\} \text {, } \\
\text { and }\left\{q_{2}, t_{2}\right\} \\
\text { consumers decide } \\
\text { whether or not to } \\
\text { purchase the platform } \\
\text { product }\end{array}$ & $\begin{array}{l}\text { Upon adopting the } \\
\text { platform product, } \\
\text { consumers decide } \\
\text { which application } \\
\text { (zero, one, or two) to } \\
\text { purchase }\end{array}$ \\
\hline
\end{tabular}

\section{ANALYSIS AND RESULTS}

In this section, we investigate the platform firm's profitability, and the provision and qualities of products in the complementary goods market under two regimes: 1) the platform firm delegates the choice of app quality to developers; and 2) the platform firm decides the specific quality level(s) by which the app developers need to abide. In the latter case, the developers are required to accept this arrangement in order to sell their products in the complementary goods market.

4.1 Pricing decisions of app developers 
We solve the model using backward-induction, by first analyzing pricing decisions of the two developers (which are made after the decision on app qualities). This assumption conforms to those in most oligopoly models, where quality is a long-run variable while prices are flexible in the short-run (see, for example, Shaked and Sutton (1982)); the intuition is that any improvement in quality might involve observable changes in long-run investments (e.g., R\&D). Given the independent decisions on $q_{1}$ and $q_{2}$, the two developers price their applications non-cooperatively by respectively solving the following:

$$
\max _{t_{i}} \bar{r}-f+q_{i}-t_{i}+q_{j}-t_{j} \quad 1-\alpha t_{i} \text { for } i=1,2 ; i \neq j
$$

where $q_{i} \geq t_{i}$. These inequalities simply reflect consumer's app purchase decisions upon adopting the platform product.

If both $t_{1}$ and $t_{2}$ are interior solutions, solving the first order conditions $\frac{\partial \pi_{i}}{\partial t_{i}}=0$ yields the optimal app prices $\left(t_{1}^{*}=t_{2}^{*}=\frac{\bar{r}-f+q_{1}+q_{2}}{3}\right)$. Note that the interior solutions require that $q_{i}>\frac{\bar{r}-f+q_{j}}{2}$ for $i=1,2 ; i \neq j$. If only one solution is interior solution (while the other is a corner solution) then equilibrium price is $t_{i}^{*}=\frac{\bar{r}-f+q_{i}}{2}$ for the interior one. The conditions that sustain this equilibrium are $q_{i}>\bar{r}-f$ and $q_{j} \leq \frac{\bar{r}-f+q_{i}}{2}$. For $q_{i} \leq \bar{r}-f$ for $i=1,2$, only corner solutions are observed in equilibrium; i.e., $t_{i}^{*}=q_{i}$ for $i=1,2$.

Two interesting observations arise from the developers' optimal pricing decisions. First, whether the presence of a particular developer contributes to the demand for the platform product (such a developer is referred to as a "demand contributor") depends on its quality level relative to that of its 
counterpart, and the surplus generated by the platform product. Second, it is possible for a developer to free-ride (such a developer is referred to as a "demand free-rider") on the positive demand generated by the other developer; however, since consumers can autonomously decide which app to purchase, the free-rider's ability to take advantage from the positive demand generated by the demand contributor and the platform firm is limited.

\subsection{Quality delegation}

Under this regime, the platform firm grants developers complete authority over the choice of quality of their apps, while setting only the price for the platform product. Consumers decide whether to purchase the platform product based on both the surplus from acquiring the platform and that from subsequent consumption of the complementary goods. We are now at the position to formally construct the objectives of the two developers in the quality development subgame.

First, suppose that $q_{1} \geq q_{2}$ (the analysis for $q_{1}<q_{2}$ is symmetric). There are three possible scenarios in light of subsequent pricing behaviors of both developers: 1) for $q_{1}>\bar{r}-f$ and $q_{2}>\frac{\bar{r}-f+q_{1}}{2}$, both developers set the same price in equilibrium (i.e., $t_{1}^{*}=t_{2}{ }^{*}=\frac{\bar{r}-f+q_{1}+q_{2}}{3}$ ) and generate positive externalities on the demand for the platform product. The equilibrium demand for the platform product as well as the two apps is characterized by $M=\bar{r}-f+q_{1}-t_{1}+q_{2}-t_{2}=\frac{\bar{r}-f+q_{1}+q_{2}}{3}$. 2) for $q_{1}>\bar{r}-f$ and $q_{2} \leq \frac{\bar{r}-f+q_{1}}{2}$, Developer 2 fully extracts the value created by its app while Developer 1 extracts only the half of the remaining consumer surplus $\left(t_{i}^{*}=\frac{\bar{r}-f+q_{i}}{2}\right)$. 3) for 
$q_{2} \leq q_{1} \leq \bar{r}-f$, both developers fully extract values they create to consumers. Therefore, Developer 1's profit function is given by:

$$
\pi_{1} q_{1}, q_{2}=\left\{\begin{array}{l}
\left(\frac{\bar{r}-f+q_{1}+q_{2}}{3}\right)^{2} 1-\alpha-\frac{1}{2} \theta_{1} q_{1}^{2} \text { if } q_{1}>\bar{r}-f \text { and } q_{2}>\frac{\bar{r}-f+q_{1}}{2} \\
\left(\frac{\bar{r}-f+q_{1}}{2}\right)^{2} 1-\alpha-\frac{1}{2} \theta_{1} q_{1}^{2} \text { if } q_{1}>\bar{r}-f \text { and } q_{2} \leq \frac{\bar{r}-f+q_{1}}{2} \\
\bar{r}-f q_{1} 1-\alpha-\frac{1}{2} \theta_{1} q_{1}^{2} \text { if } q_{2} \leq q_{1} \leq \bar{r}-f
\end{array}\right.
$$

while that of Developer 2 is:

$$
\pi_{2} q_{1}, q_{2}=\left\{\begin{array}{l}
\left(\frac{\bar{r}-f+q_{1}+q_{2}}{3}\right)^{2} 1-\alpha-\frac{1}{2} \theta_{2} q_{2}^{2} \text { if } q_{1}>\bar{r}-f \text { and } q_{2}>\frac{\bar{r}-f+q_{1}}{2} \\
\left(\frac{\bar{r}-f+q_{1}}{2}\right) q_{2} \quad 1-\alpha-\frac{1}{2} \theta_{2} q_{2}^{2} \text { if } q_{1}>\bar{r}-f \text { and } q_{2} \leq \frac{\bar{r}-f+q_{1}}{2} \\
\bar{r}-f q_{2} 1-\alpha-\frac{1}{2} \theta_{2} q_{2}^{2} \text { if } q_{2} \leq q_{1} \leq \bar{r}-f
\end{array}\right.
$$

We shall now introduce an assumption that ensures none of the developers has the incentive to produce an app of zero quality in the absence of demand externalities generated by the platform²:

Assumption 1. $\theta_{2}>\theta_{1}>\frac{1}{2}$.

Under this assumption, both profit functions are concave within their respective intervals, and are continuous in the entire domain.

To contrast the platform's profitability and quality of applications under the two regimes and highlight the effects of implementing quality control, we narrow our focus to the scenario where both

${ }^{2}$ An independent app developer's objective function can be represented by $\max _{q, t} q-t t-\frac{1}{2} \theta q^{2} \cdot q^{*}=0$ for $\theta>\frac{1}{2}$. 
demand contributor and free-rider coexist in equilibrium when the platform firm delegates quality choice to the developers. Formally, this is given by the solution to the following:

$$
\max _{q_{1}}\left(\frac{\bar{r}-f+q_{1}}{2}\right)^{2} 1-\alpha-\frac{1}{2} \theta_{1} q_{1}^{2} \text { and } \max _{q_{2}}\left(\frac{\bar{r}-f+q_{1}}{2}\right) q_{2} 1-\alpha-\frac{1}{2} \theta_{2} q_{2}^{2}
$$

that satisfies $q_{1}>\bar{r}-f$ and $q_{2} \leq \frac{\bar{r}-f+q_{1}}{2}$; and that no profitable unilateral deviation exists ${ }^{3}$. Our second assumption on developers' costs warrants that these conditions are satisfied.

Assumption 2. $\frac{1}{2}<\theta_{1}<1-\alpha<\frac{\theta_{1} 1-\alpha}{2 \theta_{1}-1-\alpha} \leq \theta_{2}$.

The equilibrium under this assumption is characterized by $q_{1}^{*}=\frac{\bar{r}-f \quad 1-\alpha}{2 \theta_{1}-1-\alpha}$ and $q_{2}{ }^{*}=\frac{\bar{r}-f 1-\alpha}{2 \theta_{1}-1-\alpha} \frac{\theta_{1}}{\theta_{2}}$. Note that $q_{1}{ }^{*}$ and $q_{2}{ }^{*}$ are multiplicative functions of $\bar{r}-f$. This implies that the pricing decision of the platform firm is not a source of cross-side network effects in equilibrium; i.e. platform's pricing does not dictate whether the high-quality app developer or low-quality app developer would assume the role of demand contributor or free rider.

Our last step in the solution process involves deriving the optimal platform price $f$ in the first stage of the game. Anticipating the subsequent quality and price choices of the developers, the platform firm's objective function is given by:

$$
\max _{f} \bar{r}-f+q_{1}-t_{1}+q_{2}-t_{2} \quad f-c+\alpha t_{1}+t_{2}
$$

${ }_{3} \theta_{2} \geq \frac{\theta_{1} 1-\alpha}{2 \theta_{1}-1-\alpha}$ ensures that, given $q_{2}{ }^{*}=\frac{\bar{r}-f 1-\alpha}{2 \theta_{1}-1-\alpha} \frac{\theta_{1}}{\theta_{2}}$, Developer 1 has no incentive to deviate to produce at a quality $q_{1}<q_{2}^{*}$. 
Substituting $q_{1}{ }^{*} f=\frac{\bar{r}-f 1-\alpha}{2 \theta_{1}-1-\alpha}, \quad t_{1}^{*} f=\frac{\theta_{1} \bar{r}-f}{2 \theta_{1}-1-\alpha}$ and $q_{2}{ }^{*} f=t_{2}{ }^{*} f=\frac{\bar{r}-f}{2 \theta_{1}-1-\alpha} \frac{1-\alpha}{\theta_{2}}$ into the objective function yields $M f-c+\alpha M t_{1}+t_{2}$; where $M=\frac{\theta_{1} \bar{r}-f}{2 \theta_{1}-1-\alpha}$ and $t_{1}+t_{2}=\frac{\theta_{1}}{2 \theta_{1}-1-\alpha}\left(1+\frac{1-\alpha}{\theta_{2}}\right) \bar{r}-f$.

Observe that a marginal increase in $f$ affects both profit streams (from sales of the platform

product and revenues shared from app sales) of the platform firm. In particular, the profit from platform sales is concave in $f$ (i.e. the profit is increasing in $M$ and decreasing in $f-c|d M / d f|$ ); while the profit from revenue-sharing decreases in $\alpha t_{1}+t_{2}|d M / d f|+\alpha M\left|\frac{d}{d f} t_{1}+t_{2}\right| . \quad \alpha M\left|\frac{d}{d f} t_{1}+t_{2}\right|$ reflects the reduced incentive in quality development, which in turns adversely affect the pricing power of app developers. The platform's total profit is concave in $f$, and its interior choice of $f$ that maximizes total profit is $f^{*}=\frac{\frac{\bar{r}+c}{2 \bar{r}}-\frac{\alpha \theta_{1}}{2 \theta_{1}-1-\alpha}\left(1+\frac{1-\alpha}{\theta_{2}}\right)}{1-\frac{\alpha \theta_{1}}{2 \theta_{1}-1-\alpha}\left(1+\frac{1-\alpha}{\theta_{2}}\right)} \bar{r}$.

We are interested in comparing the platform's profit and the equilibrium app quality between the case of zero revenue-sharing $\quad \alpha=0 \quad$ and that of an effective revenue-sharing arrangement $\quad 0<\alpha<1$. When $\alpha=0$, the only source of the platform firm's profit is the sales of its own product. Though the provision of complementary apps helps generate the demand for the platform product, the increase in demand is proportional to $\bar{r}-f$; i.e. the platform's objective function is independent of such an increase in the demand. As a result, the optimal platform price is $f_{\alpha=0}^{*}=\frac{\bar{r}+c}{2}$, which is the same as in the absence of any complementary goods. Proposition 1 summarizes the results under quality delegation. 
Proposition 1. Compared with the case of zero revenue-sharing, the platform firm charges a lower platform price and both developers produce at lower qualities. (All proofs appear the Appendix)

\subsection{Quality control}

In this section, we investigate the market dynamics when the platform firm imposes control on app quality.

By implementing an approval procedure, the platform firm can effectively impose an ex-ante quality

requirement for apps, which is ex-post verifiable. The platform firm realizes that the developers have

different production efficiencies, though unable to observe their exact production costs, and sets

predetermined quality level(s) such that apps of any other quality are rejected.

Developers would accept this arrangement and produce apps of the designated qualities as long as

doing so result in non-negative profits (i.e. the designated qualities must satisfy the individual rationality

constraints (IRs) of the developers). However, unlike in the case of quality delegation where individual

app developers observe their own marginal development cost in deciding at which quality level to produce,

the platform firm cannot distinguish between the more efficient and less efficient developers; thus

designating quality levels under information asymmetry. In other words, in addition to the IRs, the

platform needs to also accommodate incentive compatibility (IC) constraints of the developers into its

objective function.

Notice that the specific functional forms of these constraints vary depending not only on the different levels of app qualities $q_{1}, q_{2}$, but also on the surplus generated by the platform product. 
Further, the incentive for a developer to deviate and choose a quality that is designated for another developer is a function of the pricing decisions of both developers. These points will soon become clear in the next section.

4.3.1 Both developers contribute to the demand for the platform product

If the platform firm induces both developers to contribute to the demand for its product, taking into account their subsequent pricing decisions, the firm needs to designate $q_{i}>\frac{\bar{r}-f+q_{j}}{2}$ for $i=1,2 ; i \neq j$. Both developers set their prices to $t_{1}^{*}=t_{2}{ }^{*}=\frac{\bar{r}-f+q_{1}+q_{2}}{3}$, and the demands for both the platform product and apps are given by $\frac{\bar{r}-f+q_{1}+q_{2}}{3}$. The platform firm's objective is represented by:

$$
\begin{aligned}
& \max _{q_{1}, q_{2}, f} \pi_{P} \quad q_{1}, q_{2}, f=\frac{\bar{r}-f+q_{1}+q_{2}}{3}\left(f-c+2 \alpha \frac{\bar{r}-f+q_{1}+q_{2}}{3}\right) \\
& \pi_{1} q_{1}, q_{2}=\left(\frac{\bar{r}-f+q_{1}+q_{2}}{3}\right)^{2} 1-\alpha-\frac{1}{2} \theta_{1} q_{1}^{2} \geq 0 \\
& \begin{array}{ll}
\text { s.t. } & \pi_{2}, q_{2}=\left(\frac{\bar{r}-f+q_{1}+q_{2}}{3}\right)^{2} 1-\alpha-\frac{1}{2} \theta_{2} q_{2}^{2} \geq 0
\end{array} \\
& \text { and } \left.\begin{array}{l}
\left(\frac{\bar{r}-f+q_{1}+q_{2}}{3}\right)^{2}(-\alpha) \frac{1}{2} \theta_{1} q_{1}^{2} \geq\left(\frac{\bar{r}-f+2 q_{2}}{3}\right)^{2}(-\alpha) \frac{1}{2} \theta_{1} q_{2}^{2} ; \\
\left(\frac{\bar{r}-f+q_{1}+q_{2}}{3}\right)^{2}(-\alpha) \frac{1}{2} \theta_{2} q_{2}^{2} \geq\left(\frac{\bar{r}-f+2 q_{1}}{3}\right)^{2}(-\alpha) \frac{1}{2} \theta_{2} q_{1}^{2}
\end{array}\right\}
\end{aligned}
$$

As both qualities are larger than the surplus of the platform product $\bar{r}-f$, either unilateral deviation (i.e., Developer 1 chooses $q_{2}$ or Developer 2 chooses $q_{1}$ ) would still lead to both developers contributing to the platform demand. 
Lemma 1. Under the quality control regime where both developers contribute to the demand for the platform product, the implementable quality pair satisfies $q_{1} \geq q_{2}>\frac{\bar{r}-f+q_{1}}{2}$.

Independent of the platform firm's profit, incentive compatibility alone implies that the quality designated to Developer 2 (the less efficient type) cannot be higher than that designated to Developer 1 (the more efficient type). Any attainable pair of quality levels must satisfy $q_{1} \geq q_{2}$ to ensure implementability of quality control.

Since the platform firm's profit increases in both $q_{1}$ and $q_{2}$, the firm finds it desirable to require both developers to produce at as high a quality level as possible, so long as it does not violate their respective IRs. Further, since Developer 1 enjoys a relatively lower marginal development cost, the platform firm would like to take advantage of its production efficiency by designating a strictly higher quality level to this developer than that to Developer 2 (i.e., $q_{1}>q_{2}$ ). Due to information asymmetry, however, Developer 1 has the incentive to mimic its high-cost counterpart and produce a lower quality product to reduce overall development cost. Nevertheless, this imitation is also associated with a demand loss and price reduction for Developer 1 (both decrease from $\frac{\bar{r}-f+q_{1}+q_{2}}{3}$ to $\frac{\bar{r}-f+2 q_{2}}{3}$ ). Without external devices such as monetary subsidies typical used in adverse selection models, it is unclear whether the platform firm can leverage on the cross-side network effects to screen developers and how it should determine the optimal quality levels. Formally, this implies that if $q_{1}>q_{2}>\frac{\bar{r}-f+q_{1}}{2}$, then the downward IC can be simplified to: 


$$
\frac{1}{9} 2 \bar{r}-f+q_{2}+q_{1}+q_{2} \quad 1-\alpha-\frac{1}{2} \theta_{1} \quad q_{2}+q_{1} \geq 0
$$

where the first component denotes the loss in Developer 1's revenue; while the second component the gain from cost saving if it chooses to deviate to producing at $q_{2}$.

Observe that for a given $q_{1}$, the downward IC delineates the lower bound for the quality requirement on Developer 2 to $\left(q_{2} \geq \frac{q_{1} 9 \theta_{1}-21-\alpha-4 \bar{r}-f 1-\alpha}{321-\alpha-3 \theta_{1}}\right) ;{ }^{4}$ at the same time, for a given $q_{2}$, the upper bound for the quality requirement on Developer 1 also reduces to $\left(q_{1} \leq \frac{321-\alpha-3 \theta_{1} q_{2}+4 \bar{r}-f \quad 1-\alpha}{9 \theta_{1}-21-\alpha}\right) ;$ the incentive compatibility constraints require that the difference between $q_{1}$ and $q_{2}$ to be constrained from above; otherwise, the loss in demand along with the reduction in price cannot prevent the low-cost developer from behaving like its high-cost counterpart. This is an unfavorable consequence of demand complementarity among apps, because the penalty for Developer 1 to produce lower quality app is moderated by its high-cost counterpart's strategic pricing (i.e., Developer 2 also reduces its price), lessening threat more for larger difference in qualities.

Lemma 2 suggests that a separating strategy $q_{1}>q_{2} \quad$ can be implementable only when both developers enjoy relatively low marginal development cost.

\footnotetext{
4 The IC (3.7) requires $\theta_{1}<\frac{2}{3} 1-\alpha$. Otherwise, for a relatively large marginal development cost, savings from deviating and producing a lower quality product will always be attractive to Developer 1 (irrespective of the magnitude of the difference between the two designated qualities) (see details in the Appendix).
} 
Lemma 2. Under the quality control regime, inducing both developers to contribute to the demand for the platform product at different app qualities $q_{1}>q_{2}>\frac{\bar{r}-f+q_{1}}{2}$ (a separating strategy) is implementable if and only if $\theta_{1}<\frac{2}{3} 1-\alpha$ and $\theta_{2}<21-\alpha$.

This optimal solution for the separating strategy is characterized by

$$
\begin{aligned}
& q_{1}=\frac{221-\alpha-9 \theta_{1}+6 \sqrt{21-\alpha \theta_{2}} 1-\alpha \bar{r}-f}{27 \theta_{1} \sqrt{21-\alpha \theta_{2}}-21-\alpha \quad 4-4 \alpha+3 \sqrt{21-\alpha \theta_{2}}},
\end{aligned}
$$

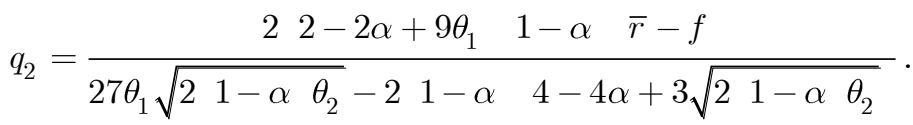

and

Facing the conflict between fostering strategic complementarity in qualities among apps (e.g.,

$\frac{\partial^{2} \pi_{1} q_{1}, q_{2}}{\partial q_{1} \partial q_{2}}>0$ and $\frac{\partial^{2} \pi_{2} q_{1}, q_{2}}{\partial q_{1} \partial q_{2}}>0$ in IRs of (3.6); a higher $q_{2}$ can sustain a higher $q_{1}$ in equilibrium or vice versa) and maintaining incentives for the efficient developer to develop a high quality product, the platform firm can simply require both developers to produce at the same quality level (a pooling strategy) and forgo the opportunity to take advantage of the production efficiency of Developer 1, thus neutralizing the incentive problem. Alternatively, it can designate a quality that is low enough for the inefficient type (Developer 2) to mitigate the effects of strategic pricing (while $\frac{\partial^{2} \pi_{1} q_{1}, q_{2}}{\partial q_{1} \partial q_{2}}=0$; details appear in 4.3.2), which leads to effectively penalizing Developer 1 for deviation. 
We shall first consider the case where the platform firm pursues a pooling strategy. Since only a uniform quality level is allowed by the platform, there is no room for deviations among app developers; in other words, both ICs become irrelevant. The platform firm's profit function is therefore:

$$
\begin{aligned}
& \max _{q, f} \frac{\bar{r}-f+2 q}{3}\left(f-c+2 \alpha \frac{\bar{r}-f+2 q}{3}\right) \\
& \text { s.t. }\left(\frac{\bar{r}-f+2 q}{3}\right)^{2} 1-\alpha-\frac{1}{2} \theta_{1} q^{2} \geq 0 \text { and }\left(\frac{\bar{r}-f+2 q}{3}\right)^{2} 1-\alpha-\frac{1}{2} \theta_{2} q^{2} \geq 0
\end{aligned}
$$

Optimality of the solution occurs when the IR condition of the high-cost developer binds while that of the low-cost developer $\left(\left(\frac{\bar{r}-f+2 q}{3}\right)^{2} 1-\alpha-\frac{1}{2} \theta_{1} q^{2} \geq 0\right)$ is slack. Therefore, the equilibrium candidate is a solution to $\left(\frac{\bar{r}-f+2 q}{3}\right)^{2} 1-\alpha-\frac{1}{2} \theta_{2} q^{2}=0$; which gives $q=\frac{41-\alpha+3 \sqrt{21-\alpha \theta_{2}}}{9 \theta_{2}-81-\alpha} \bar{r}-f$, where $q>\bar{r}-f$ requires $\theta_{2}<21-\alpha$. Taken together the cost structures identified in Lemma 2 , this implies that it is necessary for the marginal cost of Developer 2 to be relatively small to induce both developers to contribute to cross-side network effects.

Under the cost structures identified in Lemma 2, both pooling and separating strategies are implementable. In this case, we are interested in comparing these two strategies with regards to platform firm's pricing decisions and profitability. Notice that both candidates for equilibrium qualities are functions of $\bar{r}-f ;^{5}$ in other words, pricing decision of the platform firm affects provision of the apps and

\footnotetext{
${ }^{5}$ Both solutions are implied by IRs or ICs; which specific constraint binds in equilibrium depends on the property of the platform firm's profit function.
} 
their subsequent sales. For a given $f$, the overall quality $q_{1}+q_{2}$ is larger under the separating strategy than pooling strategy - in fact, both qualities in (3.8) are higher than that under the pooling strategy $\left(q=\frac{41-\alpha+3 \sqrt{21-\alpha \theta_{2}}}{9 \theta_{2}-81-\alpha} \bar{r}-f\right) .{ }^{6}$ Therefore, marginal gain for the platform firm is larger from lowering the price to motivate developers to produce at higher qualities and sharing the subsequent app sales, hence we observe the equilibrium platform price to be lower under the separating strategy. The separating strategy also leads to a higher profit. In sum, if the platform firm induces both developers to contribute to the demand for the platform product, the platform firm chooses to pursue the separating strategy whenever it is implementable (see the Appendix for details).

4.3.2 Only one developer contributes to the demand for the platform product Suppose now the platform firm induces only one developer to contribute to the demand for its product in equilibrium. Note that the shape of the platform firm's objective function depends on the relative qualities chosen by the developers, which is given by:

\footnotetext{
6 This is, again, due to that $q_{1}$ and $q_{2}$ are "strategic complements" when both developers contribute to the demand for the platform product. The binding of the IR condition for the high-cost developer, i.e., $\left(\frac{\bar{r}-f+q_{1}+q_{2}}{3}\right)^{2} 1-\alpha-\frac{1}{2} \theta_{2} q_{2}^{2}=0$
} produces a larger $q_{2}$ when $q_{1}>q_{2}$ instead of $q_{1}=q_{2}$. 


$$
\begin{aligned}
& \max _{q_{1}, q_{2}, f} \pi_{P} \quad q_{1}, q_{2}, f=\frac{\bar{r}-f+q_{1}}{2}\left(f-c+\alpha\left(\frac{\bar{r}-f+q_{1}}{2}+q_{2}\right)\right) \\
& \text { s.t. } \quad \pi_{1} q_{1}, q_{2}=\left(\frac{\bar{r}-f+q_{1}}{2}\right)^{2} 1-\alpha-\frac{1}{2} \theta_{1} q_{1}^{2} \geq 0 \\
& \qquad \pi_{2} q_{1}, q_{2}=\left(\frac{\bar{r}-f+q_{1}}{2}\right) q_{2} 1-\alpha-\frac{1}{2} \theta_{2} q_{2}{ }^{2} \geq 0 \\
& \text { IIRs }) \\
& \text { and }\left(\frac{\bar{r}-f+q_{1}}{2}\right) q_{2} \quad(-\alpha) \frac{1}{2} \theta_{2} q_{2}{ }^{2} \geq\left(\frac{\bar{r}-f+2 q_{1}}{3}\right)^{2}(-\alpha) \frac{1}{2} \theta_{2} q_{1}^{2} \quad \text { (upward IC) }
\end{aligned}
$$

The functional form of the downward IC varies based on the level of quality designated to the

high-cost developer relative to that to the low-cost one; because the developers exhibit different pricing behaviors should deviation occurs. Specifically, this IC can be written as:

$$
\begin{array}{r}
\left(\frac{\bar{r}-f+q_{1}}{2}\right)^{2} 1-\alpha-\frac{1}{2} \theta_{1} q_{1}^{2} \geq \bar{r}-f q_{2} 1-\alpha-\frac{1}{2} \theta_{1} q_{2}^{2} \quad \forall q_{2} \leq \bar{r}-f ; \\
\left(\frac{\bar{r}-f+q_{1}}{2}\right)^{2} 1-\alpha-\frac{1}{2} \theta_{1} q_{1}{ }^{2} \geq\left(\frac{\bar{r}-f+2 q_{2}}{3}\right)^{2} 1-\alpha-\frac{1}{2} \theta_{1} q_{2}{ }^{2} \quad \forall \bar{r}-f<q_{2} \leq \frac{\bar{r}-f+q_{1}}{2}
\end{array}
$$

The first inequality describes a situation where the demand contributor deviates and behaves like

a free-rider; while the second inequality describes a situation where the deviation by the demand

contributor also converts its counterpart (who was previously a free-rider) into a demand contributor.

However, the platform firm always imposes a quality requirement with $q_{2} \leq \bar{r}-f$ rather than $\bar{r}-f<q_{2} \leq \frac{\bar{r}-f+q_{1}}{2}$ on Developer 2, as by doing so the platform firm forgoes the possibility of fostering the strategic complementarity in qualities $\left(\frac{\partial^{2} \pi_{1} q_{1}, q_{2}}{\partial q_{1} \partial q_{2}}=0\right)$ while retaining the undesirable strategic 
pricing behaviors among app developers that prevents the platform firm from effectively penalizing

Developer 1's deviation.

As the platform firm's profit function is strictly increasing in the quality produced by the low-cost developer $q_{1}$, the quality level designated to the low-cost developer would be higher compared with the developer's self-interest quality level; the IC of Developer 1 (3.11), which represents the required rent due to the presence of the Developer 2 (who produces at a nonzero level), must bind. An increase in quality designated to the inefficient developer $\quad q_{2}$ always attract the efficient developer to deviate and produce at $q_{2}$. To restore incentive compatibility for Developer 1 , the platform firm needs to lower $q_{1}$ at a rate faster than it increases $q_{2}$. Moreover, increase in $q_{2}$ leads only to an increase in the shared app sales from the high-cost developer, while the associated decrease in $q_{1}$ hurts both the demand for its own product and the shared app sales from the low-cost developer. As a result, it is optimal for the platform firm to shut down the high-cost developer while relying only low-cost developer to generate the cross-side network effect (i.e., $q_{1}=\frac{1-\alpha+\sqrt{21-\alpha \theta_{1}}}{2 \theta_{1}-1-\alpha} \bar{r}-f \quad$ and $q_{2}=0$ ). The following lemma summarizes these points. 
Lemma 3. Under the quality control regime, the platform firm that induces only the low-cost developer to contribute to the cross-side network effects shuts down the high-cost developer unless $\theta_{1}>z$

7 and $\theta_{2}<\frac{2 \sqrt{1-\alpha}+{\sqrt{2 \theta_{1}}}^{2}}{9}$.

Under these specific cost structures present in Lemma 3, Developer 2, however, finds it optimal to deviate and produce at $q_{1}$, capturing a strictly positive rent. This case is known as "countervailing incentives" (Laffont and Martimont 2002), where the incentive constraint of the inefficient developer (i.e., the upward IC) binds in equilibrium. In these cases, shutting down Developer 2 is no longer implementable. The new implementable pair satisfying $q_{1}>\bar{r}-f$ and $0<q_{2} \leq \bar{r}-f$ is therefore the one that binds both the downward and upward ICs, and it implies less profit for the platform firm than $q_{1}=\frac{1-\alpha+\sqrt{21-\alpha \theta_{1}}}{2 \theta_{1}-1-\alpha} \bar{r}-f$ and $q_{2}=0$ does.

While Lemmas 2 and 3 suggest that different qualities might be observed in equilibria, our next proposition concludes that it is optimal for the platform firm to induce the production of apps of a single quality in the complementary goods market.

Proposition 2. Under the quality control regime, the platform firm finds it optimal to either impose the same quality requirement on the developers (a pooling strategy), with both contributing to the demand for

7 The specific form of $z \in\left(\frac{1}{2}, 1-\alpha\right)$ is lengthy and thus delegated to the Appendix. 
the platform product; or to induce only the low-cost (i.e., more efficient) developer to participate while shutting down the high-cost developer.

If shutting down the inefficient developer is not feasible due to "countervailing incentives" when marginal development costs of the two developers are relatively close, it is always more profitable for the platform firm to switch to the pooling strategy with both developers contributing to the demand for its product. On the other hand, when the efficient developer enjoys a significant cost advantage, even though the platform firm's profit under the separating strategy dominates that under the pooling strategy (discussion following lemma 2), it is still inferior to shutting down the high-cost developer under the same cost structure, as it is too costly for the platform firm to reserve the incentive compatibility (by constraining the quality designated to Developer 1 and thus underexploiting this cost advantage) given the strategic pricing exist between the two apps. Proposition 2 thus follows the fact that both scenarios with different qualities are dominated either by the pooling case or by the shut-down case. Moreover, both single-quality scenarios can be optimal depending on the pair of marginal development costs.

\subsection{Comparison between quality delegation and quality control}

Having established the previous propositions, we can now analyze the platform firm's profitability under quality delegation vs. quality control. We found that two quality levels exist in equilibrium under the quality delegation regime, while only one level is possible under quality control. Further, in some cases the inefficient developer would be shut down under the quality control regime, thereby lessening the 
variety of apps available on the platform. While these observations appear to suggest that leaving developers autonomy in their quality choice is beneficial to the platform firm, our next proposition indicates that the opposite is indeed more desirable for the platform firm.

Proposition 3. The quality control regime always results in higher profit for the platform firm compared with the case of quality delegation.

For conditions that allow the platform firm to induce both developers to contribute to the demand for the platform product, centralizing the quality decision allows the platform firm to foster strategic complementarity in qualities between the two app developers. Though in the pooling equilibrium under quality control, the efficient developer may produces at a lower quality level than under the quality delegation regime (due to information asymmetry faced by the platform firm), the negative effects are offset by converting the original demand free-rider (Developer 2) into a demand contributor.

It is perhaps surprising to see that shutting down the inefficient type and forgoing the product variety in the complementary goods market under the quality control regime still outperforms accommodating two apps under the quality delegation regime. Notice, however, that shutting down the inefficient developer occurs when the inefficient developer has a relatively high marginal cost; given Developer 2's high marginal development cost, under quality delegation, Developer 1 anticipates that it would be the only demand contributor at equilibrium and reduces its incentives to develop high quality 
app in the first place. These forces together drive down the demand and profit for the platform. In sum, delegating the choice to more informed agents does not necessarily benefit the platform firm.

\section{CONCLUSION}

The contributions of this work with regards to extant literature on two-sided markets are two-fold: First, by relaxing the assumption that the presence or number of participants in one side of the market always generate positive externalities to the other side of the market, our analysis unveils the complex dynamics underlying the strategic considerations by the platform firm and developers of complementary products. This allows us to capture the cross-side externalities observed in real markets. Second, ours is one of the first attempts to explicitly model incentives of providers of complementary products to develop high-quality goods, and how a developer's decision might be influenced by both the response of the other developer and the platform, thereby making concrete recommendations to the platform firm on the optimal intervention and pricing strategies. In the absence of both search cost on the consumer side and competition among app developers, our analysis provides an additional explanation on why platform firms might want to intervene the complementary goods market.

Our results also carry significant implications to the current mobile platform market. For example, Apple is known for its rigorous approval process on apps and sharing revenues from app sales, while Google adopts a "hands-off" approach in the Android market without quality control or revenue-sharing with app developers. Our results suggest that controlling quality of complementary 
goods is indeed beneficial to the platform firm, and that it is important to reduce the variance in quality

levels. The underlying rationale is that, by allowing only a uniform quality level, the platform firm can

leverage on both developers' contributions to the demand for the platform product or from shutting-down

the inefficient developer; while the cross-side network effects suggest that it may be too costly to deliver information rents to the more efficient developer from screening the qualities of the complementary

products when they are significantly different.

\section{REFERENCE}

Armstrong, M. 2006. Competition in Two-Sided Markets. The RAND Journal of Economics 37668-691.

Bakos, Y. and E. Katsamakas. 2008. Design and Ownership of Two-Sided Networks: Implications for Internet Platforms. Journal of Management Information Systems 25(2)171-202.

Boudreau, K. 2010. Open Platform Strategies and Innovation: Granting Access vs. Devolving Control. Management Science 561849-1872.

Caillaud, B. and B. Jullien. 2003. Chicken \& Egg: Competition among Intermediation Service Providers. Rand Journal of Economics 34309-328.

Economides, N. and E. Katsamakas. (2006). Two-Sided Competition of Proprietary vs. Open Source Technology Platforms and the Implications for the Software Industry. Management Science, 52(7), $1057-1071$.

Hagiu, A. 2006. Pricing and Commitment by Two-Sided Platforms. The RAND Journal of Economics 37720-737.

Hagiu, A. 2009. Two-Sided Platforms: Product Variety and Pricing Structures. Journal of Economics \& Management Strategy 181011-1043.

Katz, M. L. and C. Shapiro. 1994. Systems Competition and Network Effects. The Journal of Economic Perspectives 893-115.

Lin, M., Li, S., and A. B. Whinston. (2011). Innovation and Price Competition in a Two-Sided Market. Journal of Management Information Systems, 28(2), 171 - 202. 
Rochet, J.-C. and J. Tirole. 2006. Two-Sided Markets: A Progress Report. Rand Journal of Economics $37(3) 645-667$.

Shaked, A. and J. Sutton. 1982. Relaxing Price Competition Through Product Differentiation. Review of Economic Studies 493-13. 


\section{Appendix}

\section{Proof of Proposition 1:}

The platform's total profit is concave in $f$, and its choice of $f$ that maximizes total profit is

$f^{*}=\max \left\{\frac{\frac{\bar{r}+c}{2 \bar{r}}-\frac{\alpha \theta_{1}}{2 \theta_{1}-1-\alpha}\left(1+\frac{1-\alpha}{\theta_{2}}\right)}{1-\frac{\alpha \theta_{1}}{2 \theta_{1}-1-\alpha}\left(1+\frac{1-\alpha}{\theta_{2}}\right)} \bar{r}, c\right\}$. The corresponding equilibrium is characterized by
$q_{1}{ }^{*}=\frac{\frac{\bar{r}-c}{2} 1-\alpha}{2 \theta_{1}-1-\alpha-\alpha \theta_{1}\left(1+\frac{1-\alpha}{\theta_{2}}\right)}, q_{2}{ }^{*}=\frac{\theta_{1}}{\theta_{2}} q_{1}{ }^{*}$, and $\pi_{P}{ }^{*}=\frac{\bar{r}-c^{2} \theta_{1} \theta_{2}}{41-\alpha+\alpha-2 \theta_{1} \theta_{2}+41-\alpha \alpha \theta_{1}}$ if $f^{*}$

is the interior solution ; and $q_{1}^{*}=\frac{\bar{r}-c 1-\alpha}{2 \theta_{1}-1-\alpha}, q_{2}^{*}=\frac{\theta_{1}}{\theta_{2}} q_{1}{ }^{*}$, and $\pi_{P}^{*}=\alpha\left(1+\frac{1-\alpha}{\theta_{2}}\right)\left(\frac{\theta_{1} \bar{r}-c}{2 \theta_{1}-1-\alpha}\right)^{2}$ if

$f^{*}=c$.

Since $\frac{\partial q_{1}^{*}}{\partial \alpha}=\frac{\left(\frac{1-\alpha^{2}}{\theta_{2}}-1\right) \theta_{1}}{\left(2 \theta_{1}-1-\alpha-\alpha \theta_{1}\left(1+\frac{1-\alpha}{\theta_{2}}\right)\right)^{2}} \frac{\bar{r}-c}{2}<0$ if $f^{*}$ is the interior solution and

$\frac{\partial q_{1}^{*}}{\partial \alpha}=\frac{-2 \theta_{1}}{2 \theta_{1}-1-\alpha^{2}} \bar{r}-c<0$ if $f^{*}=c$, nonzero revenue sharing leads to lower qualities from both

app developers.

Comparing $f^{*}=\max \left\{\frac{\frac{\bar{r}+c}{2 \bar{r}}-\frac{\alpha \theta_{1}}{2 \theta_{1}-1-\alpha}\left(1+\frac{1-\alpha}{\theta_{2}}\right)}{1-\frac{\alpha \theta_{1}}{2 \theta_{1}-1-\alpha}\left(1+\frac{1-\alpha}{\theta_{2}}\right)} \bar{r}, c\right\}$ with $f_{\alpha=0}^{*}=\frac{\bar{r}+c}{2}$ yields $f^{*}<f_{\alpha=0}^{*}$

for $\frac{\alpha \theta_{1}}{2 \theta_{1}-1-\alpha}\left(1+\frac{1-\alpha}{\theta_{2}}\right)$. 


\section{Proof of Lemma 1:}

Suppose on the contrary $q_{2}>q_{1},\left(\frac{\bar{r}+q_{1}+q_{2}-f}{3}\right)^{2} 1-\alpha-\frac{1}{2} \theta_{1} q_{1}^{2} \geq\left(\frac{\bar{r}+2 q_{2}-f}{3}\right)^{2} 1-\alpha-\frac{1}{2} \theta_{1} q_{2}^{2}$ can be simplified to $\frac{1}{9} \bar{r}+q_{1}+q_{2}-f+\bar{r}+2 q_{1}-f \quad 1-\alpha \geq \frac{1}{2} \theta_{2} \quad q_{2}+q_{1}$

and $\left(\frac{\bar{r}+q_{1}+q_{2}-f}{3}\right)^{2} 1-\alpha-\frac{1}{2} \theta_{1} q_{1}^{2} \geq\left(\frac{\bar{r}+2 q_{2}-f}{3}\right)^{2} 1-\alpha-\frac{1}{2} \theta_{1} q_{2}^{2}$ can be simplified to

$\frac{1}{2} \theta_{1} q_{2}+q_{1} \geq \frac{1}{9} 2 \bar{r}+q_{2}-f+q_{1}+q_{2} \quad 1-\alpha$. Adding these two terms yields

$\frac{2}{9} q_{1}-q_{2} \quad 1-\alpha \geq \frac{1}{2} \theta_{2}-\theta_{1} \quad q_{2}+q_{1} . \quad$ A contradiction occurs since $\theta_{2}>\theta_{1}$.

\section{Proof of Lemma 2:}

For $\theta_{1} \geq \frac{2}{3} 1-\alpha, \frac{1}{9} 2 \bar{r}-f+q_{2}+q_{1}+q_{2} \quad 1-\alpha-\frac{1}{2} \theta_{1} q_{2}+q_{1}<0$ given $q_{1}>q_{2}>\bar{r}-f$,

implies that the IC is always violated. Hence for a relatively large marginal development cost, savings

from deviating and producing a lower quality product will always be attractive to Developer 1

(irrespective of the magnitude of the difference between the two designated qualities). Therefore, it is

necessary for Developer 1 to enjoy a sufficiently low marginal development cost, $\theta_{1}<\frac{2}{3} 1-\alpha$.

Since the platform firm's objective function $\frac{\bar{r}-f+q_{1}+q_{2}}{3}\left(f-c+2 \alpha \frac{\bar{r}-f+q_{1}+q_{2}}{3}\right)$ is increasing in both $q_{1}$ and $q_{2}$, the optimal $q_{1}$ and $q_{2}$ are achieved when some of the constraints bind. To solve this optimization problem with nonlinear constraints, we first drop the upward IC condition and re-examine it later. 
The IR condition of Developer $1, \pi_{1} q_{1}, q_{2}=\left(\frac{\bar{r}-f+q_{1}+q_{2}}{3}\right)^{2} 1-\alpha-\frac{1}{2} \theta_{1} q_{1}^{2} \geq 0$, is concave in $q_{1}$. It can be simplified to

$q_{1} \leq \frac{\sqrt{21-\alpha} q_{2}+\bar{r}-f}{3 \sqrt{\theta_{1}}-\sqrt{21-\alpha}}=\frac{q_{2}+\bar{r}-f \quad 3 \sqrt{21-\alpha \theta_{1}}+21-\alpha}{9 \theta_{1}-21-\alpha} . \quad$ The downward IC condition implies that $q_{1} \leq \frac{321-\alpha-3 \theta_{1} q_{2}+41-\alpha \quad \bar{r}-f}{9 \theta_{1}-21-\alpha}$

For a given $q_{2}$, the platform firm chooses the maximum $q_{1}$ that simultaneously satisfies both inequalities.

Which inequality would bind in equilibrium depends on the relative magnitude of

$\frac{q_{2}+\bar{r}-f \quad 3 \sqrt{21-\alpha \theta_{1}}+21-\alpha}{9 \theta_{1}-21-\alpha}$ and $\frac{321-\alpha-3 \theta_{1} q_{2}+4 \bar{r}-f 1-\alpha}{9 \theta_{1}-21-\alpha}$. Since

$\frac{q_{2}+\bar{r}-f \quad 3 \sqrt{21-\alpha \theta_{1}}+21-\alpha}{9 \theta_{1}-21-\alpha} \geq \frac{321-\alpha-3 \theta_{1} q_{2}+4 \bar{r}-f 1-\alpha}{9 \theta_{1}-21-\alpha}$ implies that

$3 \sqrt{21-\alpha \theta_{1}}+9 \theta_{1}-41-\alpha \quad q_{2} \geq \sqrt{21-\alpha} \sqrt{21-\alpha}-3 \sqrt{\theta_{1}} \bar{r}-f$, which is true for any $q_{2}>0$ and $\bar{r}-f>0 ; 3 \sqrt{21-\alpha \theta_{1}}+9 \theta_{1}-41-\alpha>0$ and $\sqrt{21-\alpha}-3 \sqrt{\theta_{1}}<0$ for $\theta_{1}<\frac{2}{3} 1-\alpha$. Hence, it can be concluded that the downward IC condition binds instead of the IR condition for the low-cost developer.

According to the IR of the high-cost developer, $\frac{1}{2} \bar{r}+q_{1}-f<q_{2} \leq \frac{21-\alpha+3 \sqrt{21-\alpha \theta_{2}}}{9 \theta_{1}-21-\alpha}+q_{1}-f$.

For a given $q_{1}$, the platform firm chooses the maximum $q_{2}$; which is the solution to $q_{2}=\frac{21-\alpha+3 \sqrt{21-\alpha \theta_{2}}}{9 \theta_{1}-21-\alpha} \bar{r}+q_{1}-f$ 
In sum, $q_{2}=\frac{21-\alpha+3 \sqrt{21-\alpha \theta_{2}}}{9 \theta_{1}-21-\alpha} \bar{r}+q_{1}-f$ and $q_{1}=\frac{321-\alpha-3 \theta_{1} q_{2}+41-\alpha \bar{r}-f}{9 \theta_{1}-21-\alpha}$ together determine $q_{1}$ and $q_{2}$. The equilibrium is characterized by (3.8) in the main text where $27 \theta_{1} \sqrt{21-\alpha \theta_{2}}-21-\alpha \quad 4-4 \alpha+3 \sqrt{21-\alpha \theta_{2}}>0$ for $\theta_{1}<\frac{2}{3} 1-\alpha$ and $\theta_{2} \geq \frac{\theta_{1}}{2 \theta_{1}-1+\alpha} 1-\alpha>1-\alpha . \quad$ Indeed, $q_{1}^{*}>\bar{r}-f$ for $\theta_{1}<\frac{2}{3} 1-\alpha \quad$ (which is verified by simplifying the inequality given by $\frac{221-\alpha-9 \theta_{1}+6 \sqrt{21-\alpha \theta_{2}}}{27 \theta_{1} \sqrt{21-\alpha \theta_{2}}-21-\alpha 4-4 \alpha+3 \sqrt{21-\alpha \theta_{2}}}-\alpha>1$; the simplified inequality is $21-\alpha+3 \sqrt{21-\alpha \theta_{2}} \quad 21-\alpha-3 \theta_{1}>0$ for $\left.\theta_{1}<\frac{2}{3} 1-\alpha\right)$. This implies that Developer 1 would assume the role as a demand contributor required in equilibrium.

Finally, we check whether dropping the upward IC would affect the equilibrium solution. Given $q_{1}>q_{2}$, the upward IC condition, $\left(\frac{\bar{r}-f+q_{1}+q_{2}}{3}\right)^{2} 1-\alpha-\frac{1}{2} \theta_{2} q_{2}{ }^{2} \geq\left(\frac{\bar{r}-f+2 q_{1}}{3}\right)^{2} 1-\alpha-\frac{1}{2} \theta_{2} q_{1}^{2}$, can be simplified to $-\frac{1}{9} 2 \bar{r}-f+q_{1}+q_{1}+q_{2} \quad 1-\alpha+\frac{1}{2} \theta_{2} \quad q_{2}+q_{1} \geq 0$.

Substituting two qualities in (3.8) into the upward IC yields

$$
\frac{241-\alpha \sqrt{21-\alpha \theta_{2}}+3 \theta_{2} 21-\alpha+3 \sqrt{21-\alpha \theta_{2}}-3 \theta_{1} 3 \sqrt{21-\alpha \theta_{2}}-21-\alpha}{327 \theta_{1} \sqrt{21-\alpha \theta_{2}}-21-\alpha 4-4 \alpha+3 \sqrt{21-\alpha \theta_{2}}} 1-\alpha \quad \bar{r}-f,
$$

which is strictly positive for $\theta_{1}<\frac{2}{3} 1-\alpha$ and $\theta_{2} \geq \frac{\theta_{1}}{2 \theta_{1}-1+\alpha} 1-\alpha$. Therefore, the upward IC condition is slack.

Note that when $\theta_{2} \geq \frac{\theta_{1}}{2 \theta_{1}-1+\alpha} 1-\alpha \quad$ and $\theta_{1}<\frac{2}{3} 1-\alpha, q_{1}>q_{2} \quad\left(q_{1}>q_{2}\right.$ means that $\sqrt{21-\alpha \theta_{2}}>3 \theta_{1}$; and $\sqrt{21-\alpha \theta_{2}} \geq \sqrt{21-\alpha \frac{\theta_{1}}{2 \theta_{1}-1+\alpha} 1-\alpha}=1-\alpha \sqrt{\frac{2 \theta_{1}}{2 \theta_{1}-1+\alpha}}>3 \theta_{1}$ 
where the first inequality follows $\theta_{2} \geq \frac{\theta_{1}}{2 \theta_{1}-1+\alpha} 1-\alpha$ and the second follows $\left.\theta_{1}<\frac{2}{3} 1-\alpha\right)$.

Moreover, the condition $q_{2}>\frac{1}{2} \bar{r}-f+q_{1}$ implies $\theta_{2}<21-\alpha$.

Platform firm's profit and price under the separating and pooling strategies:

Substituting $q=\frac{41-\alpha+3 \sqrt{21-\alpha \theta_{2}}}{9 \theta_{2}-8+8 \alpha} \bar{r}-f \quad$ into $\frac{\bar{r}-f+2 q}{3}\left(f-c+2 \alpha \frac{\bar{r}-f+2 q}{3}\right)$ yields

$\frac{3 \theta_{2}+2 \sqrt{21-\alpha \theta_{2}}}{9 \theta_{2}-8+8 \alpha} \bar{r}-f\left(f-c+2 \alpha \frac{3 \theta_{2}+2 \sqrt{21-\alpha \theta_{2}}}{9 \theta_{2}-8+8 \alpha} \bar{r}-f\right) ;$

Analogously, substituting $q_{1}$ and $q_{2}$ in (3.8) into the platform firm's profit function yields

$$
\begin{aligned}
& \frac{2-2 \alpha+9 \theta_{1} \sqrt{21-\alpha \theta_{2}}}{27 \theta_{1} \sqrt{21-\alpha \theta_{2}}-21-\alpha \quad 4-4 \alpha+3 \sqrt{21-\alpha \theta_{2}}} \\
& \bar{r}-f\left[f-c+\frac{2 \alpha 2-2 \alpha+9 \theta_{1} \sqrt{21-\alpha \theta_{2}}}{27 \theta_{1} \sqrt{21-\alpha \theta_{2}}-21-\alpha \quad 4-4 \alpha+3 \sqrt{21-\alpha \theta_{2}}} \bar{r}-f\right]
\end{aligned}
$$

Both are concave functions of $f$ for $\theta_{1}<\frac{2}{3} 1-\alpha$ and $\theta_{2}<21-\alpha$. The solutions to both first-order conditions w.r.t. $f$ gives $f_{\text {Pooling }}=\frac{\frac{c+\bar{r}}{2 \bar{r}}-\frac{2 \alpha 3 \theta_{2}+2 \sqrt{21-\alpha \theta_{2}}}{9 \theta_{2}-8+8 \alpha}}{1-\frac{2 \alpha 3 \theta_{2}+2 \sqrt{21-\alpha \theta_{2}}}{9 \theta_{2}-8+8 \alpha}} \bar{r} \in\left(c, \frac{c+\bar{r}}{2 \bar{r}}\right)$ (under the pooling

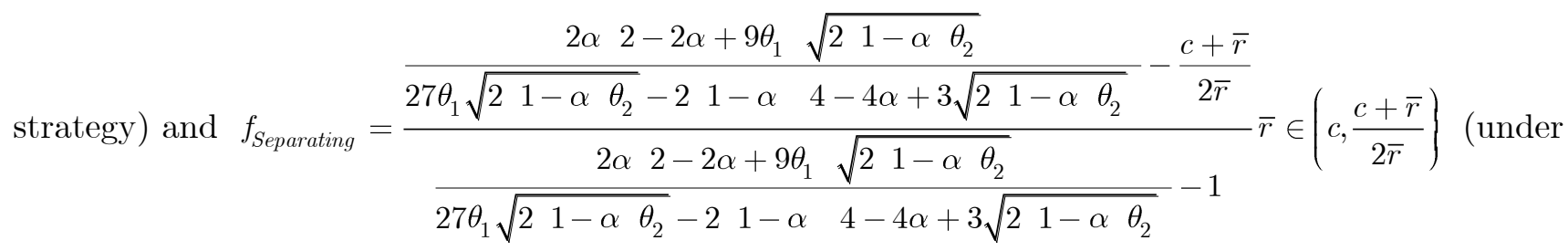
the separating strategy). Comparing the profits under the two strategies, 


$$
\begin{aligned}
& \pi_{f} \quad x=\max _{f \geq c} x \bar{r}-f \quad f-c+2 x \bar{r}-f \alpha \\
& =f^{2} x-1+2 x \alpha+f x \quad c+\bar{r}-4 x \alpha \bar{r}+x-c \bar{r}+2 x \alpha \bar{r}^{2} \\
& \stackrel{f=-\frac{c+\bar{r}-4 x \alpha \bar{r}}{2-1+2 x \alpha}}{\longrightarrow} \frac{1}{4} \frac{x \bar{r}-c^{2}}{1-2 x \alpha}
\end{aligned}
$$

where $x$ denotes $\frac{3 \theta_{2}+2 \sqrt{21-\alpha \theta_{2}}}{9 \theta_{2}-8+8 \alpha}$ or $\frac{2-2 \alpha+9 \theta_{1} \sqrt{21-\alpha \theta_{2}}}{27 \theta_{1} \sqrt{21-\alpha \theta_{2}}-21-\alpha 4-4 \alpha+3 \sqrt{21-\alpha \theta_{2}}}$.

The first order derivative w.r.t. $x$ is $\frac{1-2 x \alpha+2 \alpha x}{1-2 x \alpha^{2}}=\frac{1}{1-2 x \alpha^{2}}>0$.

Since $\frac{2-2 \alpha+9 \theta_{1} \sqrt{21-\alpha \theta_{2}}}{27 \theta_{1} \sqrt{21-\alpha \theta_{2}}-21-\alpha \quad 4-4 \alpha+3 \sqrt{21-\alpha \theta_{2}}}>\frac{3 \theta_{2}+2 \sqrt{21-\alpha \theta_{2}}}{9 \theta_{2}-8+8 \alpha}, f_{\text {Separating }}<f_{\text {Pooling }}$, while the platform firm prefers the separating strategy to the pooling strategy.

For other parameter values falling in that specified by Assumption 2 and $\theta_{2}<21-\alpha$,

$f_{\text {Pooling }}=\max \left\{c, \frac{\frac{c+\bar{r}}{2 \bar{r}}-\frac{2 \alpha 3 \theta_{2}+2 \sqrt{21-\alpha \theta_{2}}}{9 \theta_{2}-8+8 \alpha}}{1-\frac{2 \alpha 3 \theta_{2}+2 \sqrt{21-\alpha \theta_{2}}}{9 \theta_{2}-8+8 \alpha}} \bar{r}\right\}$. When the platform price $f$ is an interior solution,

the corresponding profit is

$$
\frac{\theta_{2} \bar{r}-c^{2}}{43-2 \alpha \theta_{2}-8 \sqrt{21-\alpha \theta_{2}}} \text { and } q=\frac{41-\alpha+3 \sqrt{21-\alpha \theta_{2}}}{29-6 \alpha \theta_{2}-8+4 \alpha 2-\sqrt{21-\alpha \theta_{2}}} \bar{r}-c \text {. }
$$

When the platform price $f$ is a corner solution, the corresponding profit is

$$
2 \alpha\left[\frac{3 \theta_{2}+2 \sqrt{21-\alpha \theta_{2}} \bar{r}-c}{9 \theta_{2}-8+8 \alpha}\right]^{2} \text { and } q=\frac{41-\alpha+3 \sqrt{21-\alpha \theta_{2}}}{9 \theta_{2}-8+8 \alpha} \bar{r}-c
$$

\section{Proof of Lemma 3:}


We first ignore the upward IC condition and the IR condition of the high-cost developer.

It is obvious that $\left(\frac{\bar{r}-f+q_{1}}{2}\right)^{2} 1-\alpha-\frac{1}{2} \theta_{1} q_{1}^{2} \geq \bar{r}-f q_{2} 1-\alpha-\frac{1}{2} \theta_{1} q_{2}^{2}$ for $q_{1}=q_{2} \geq 0$.

The LHS of the downward IC condition is

$$
\begin{aligned}
& \left(\frac{\bar{r}+q_{1}-f}{2}\right)^{2} 1-\alpha-\frac{1}{2} \theta_{1} q_{1}{ }^{2} \\
& =-\frac{1}{2}\left(\theta_{1}-\frac{1}{2} 1-\alpha\right)\left(q_{1}-\frac{\bar{r}-f 1-\alpha}{2 \theta_{1}-1-\alpha}\right)^{2}+\left(\frac{1-\alpha^{2}}{2 \theta_{1}-1-\alpha}+1-\alpha\right) \frac{1}{4} \bar{r}-f^{2}, \text { while the RHS of the }
\end{aligned}
$$

downward IC condition is $\bar{r}-f q_{2} 1-\alpha-\frac{1}{2} \theta_{1} q_{2}{ }^{2}=-\frac{1}{2} \theta_{1}\left(q_{2}-\frac{\bar{r}-f 1-\alpha}{\theta_{1}}\right)^{2}+\frac{\bar{r}-f^{2} 1-\alpha^{2}}{2 \theta_{1}}$. It can be easily verified that $\left(\frac{1-\alpha^{2}}{2 \theta_{1}-1-\alpha}+1-\alpha\right) \frac{1}{4} \geq \frac{1-\alpha^{2}}{2 \theta_{1}}$ for $1-\alpha>\theta_{1}>\frac{1}{2}$ and $0<\alpha<1$.

For $\theta_{1}<1-\alpha$, the axes of symmetry of both parabolae are larger than $\bar{r}-f\left(\frac{1-\alpha}{\theta_{1}}>1\right.$ and $\left.\frac{1-\alpha}{2 \theta_{1}-1-\alpha}>1\right)$. It implies that the RHS of the downward IC condition is positive for $q_{2} \leq \bar{r}-f$.

Therefore, the IR condition of the low-cost developers is always slack.

Since the platform firm's profit function is increasing in $q_{1}$, for a given $0<q_{2} \leq \bar{r}-f$, the downward IC condition binds at the equilibrium, i.e., $\left(\frac{\bar{r}+q_{1}-f}{2}\right)^{2} 1-\alpha-\frac{1}{2} \theta_{1} q_{1}{ }^{2}=\bar{r}-f q_{2} 1-\alpha-\frac{1}{2} \theta_{1} q_{2}{ }^{2}$. The plausible $q_{1}$ is the one intersecting the parabola on the RHS of its axis of symmetry, and is given by $q_{1}=\frac{\bar{r}-f 1-\alpha+\sqrt{\left[\begin{array}{ll}\bar{r}-f & 1-\alpha\end{array}\right]^{2}+2 \theta_{1}-1-\alpha \quad 1-\alpha \bar{r}-f^{2}-4 \bar{r}-f \quad 1-\alpha q_{2}+2 \theta_{1} q_{2}^{2}}}{2 \theta_{1}-1-\alpha}$. 
As $\frac{\bar{r}-f 1-\alpha}{\theta_{1}}>\bar{r}-f$, it is clear that $q_{1}>\bar{r}-f$. It is a function of both $q_{2}$ and $f$. The partial derivative of the platform firm's profit function, i.e., $\frac{\bar{r}-f+q_{1}}{2}\left(f-c+\alpha\left(\frac{\bar{r}-f+q_{1}}{2}+q_{2}\right)\right)$ w.r.t. $q_{2}$ is $\frac{1}{2} f+\alpha \bar{r}-f+q_{1}+q_{2}-c \frac{\partial q_{1}}{\partial q_{2}}+\frac{\bar{r}-f+q_{1}}{2} \alpha$

Applying the implicit function theorem to $\left(\frac{\bar{r}+q_{1}-f}{2}\right)^{2} 1-\alpha-\frac{1}{2} \theta_{1} q_{1}^{2}=\bar{r}-f q_{2} 1-\alpha-\frac{1}{2} \theta_{1} q_{2}{ }^{2}$, we get $\frac{\partial q_{1}}{\partial q_{2}}=\frac{\bar{r}-f 1-\alpha-\theta_{1} q_{2}}{\frac{1}{2} \bar{r}-f+q_{1} \quad 1-\alpha-\theta_{1} q_{1}}=\frac{2\left[\bar{r}-f 1-\alpha-\theta_{1} q_{2}\right]}{\bar{r}-f 1-\alpha-2 \theta_{1}-1-\alpha q_{1}}$

Substituting

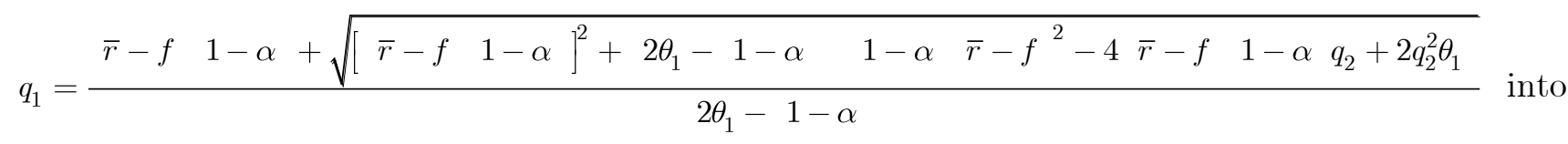
$\frac{\partial q_{1}}{\partial q_{2}}=\frac{2\left[\bar{r}-f 1-\alpha-\theta_{1} q_{2}\right]}{\bar{r}-f 1-\alpha-2 \theta_{1}-1-\alpha \quad q_{1}}$ yields

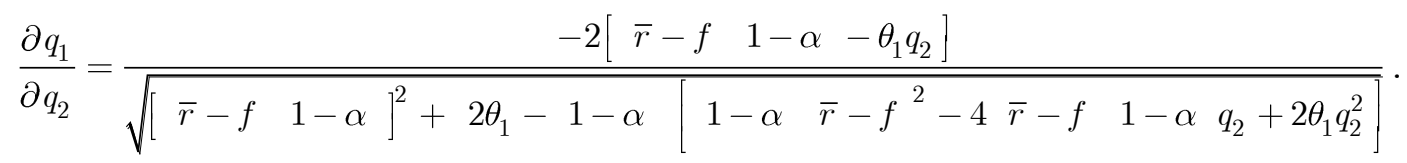

Comparing the denominator with the nominator yields

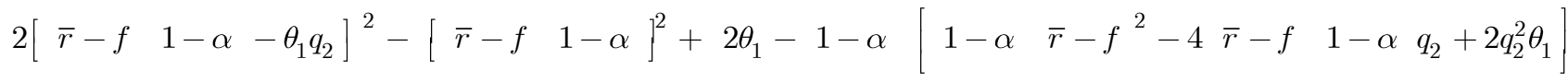

$$
\begin{aligned}
& =21-\alpha \quad \bar{r}-f-q_{2}\left[\begin{array}{llll}
2 & 1-\alpha & \bar{r}-f-\bar{r}-f+q_{2} & \theta_{1}
\end{array}\right] \geq 0
\end{aligned}
$$

in which the inequality follows $0<q_{2} \leq \bar{r}-f$ and $\theta_{1}<1-\alpha$. Therefore, $\frac{\partial q_{1}}{\partial q_{2}} \leq-1$.

As a result, $\frac{1}{2} f-c+\alpha \bar{r}-f+q_{1}+q_{2} \frac{\partial q_{1}}{\partial q_{2}}+\frac{\bar{r}-f+q_{1}}{2} \alpha<0$ for $f \geq c .^{8}$

\footnotetext{
${ }^{8}$ This rules out the possibility that the platform firm sacrifices revenue from its own product to subsidize the complementary goods market.
} 
It implies that $q_{2}=0$ is the candidate for the optimality. Accordingly, $q_{1}=\frac{1-\alpha+\sqrt{21-\alpha \theta_{1}}}{2 \theta_{1}-1-\alpha} \bar{r}-f$.

Note that when $q_{2}=0$, the low-cost developer's IR condition coincides with the downward IC condition.

It suggests that the IR condition of the high-cost developer, i.e. $\left(\frac{\bar{r}+q_{1}-f}{2}\right) q_{2} 1-\alpha-\frac{1}{2} \theta_{2} q_{2}{ }^{2} \geq 0$, is irrelevant to the optimization problem.

Now let us revisit the upward IC condition,

$\left(\frac{\bar{r}-f+q_{1}}{2}\right) q_{2} 1-\alpha-\frac{1}{2} \theta_{2} q_{2}{ }^{2}=0 \geq\left(\frac{\bar{r}-f+2 q_{1}}{3}\right)^{2} 1-\alpha-\frac{1}{2} \theta_{2} q_{1}{ }^{2}$

Under some cost structures, $\left(\frac{\bar{r}-f+2 q_{1}}{3}\right)^{2} 1-\alpha-\frac{1}{2} \theta_{2} q_{1}^{2}>0$ with $q_{1}=\frac{1-\alpha+\sqrt{21-\alpha \theta_{1}}}{2 \theta_{1}-1-\alpha}-f \quad$ and $q_{2}=0$, implying that $q_{1}=\frac{1-\alpha+\sqrt{21-\alpha \theta_{1}}}{2 \theta_{1}-1-\alpha} \bar{r}-f$ and $q_{2}=0$ is no longer implementable.

Substituting $\quad q_{1}=\frac{1-\alpha+\sqrt{21-\alpha \theta_{1}}}{2 \theta_{1}-1-\alpha} \bar{r}-f \quad$ and $\quad q_{2}=0$ into $\left(\frac{\bar{r}-f+2 q_{1}}{3}\right)^{2} 1-\alpha-\frac{1}{2} \theta_{2} q_{1}^{2}$ yields $\frac{21-\alpha 1-\alpha+2 \theta_{1}+2{\sqrt{21-\alpha \theta_{1}}}^{2}-91-\alpha+{\sqrt{21-\alpha \theta_{1}}}^{2} \theta_{2}}{182 \theta_{1}-1-\alpha^{2}}-f^{2}$.

It is strictly positive when

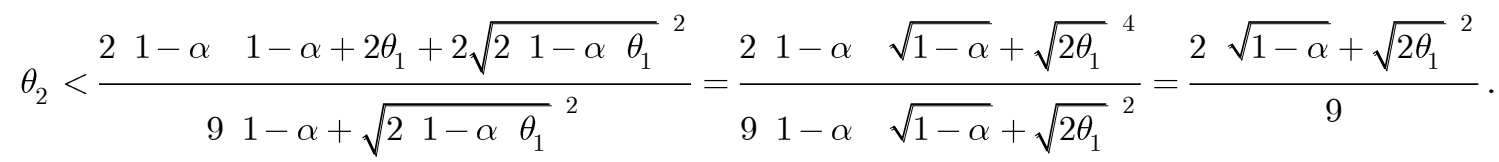

Note that for $\theta_{1}$ that makes $\frac{2 \sqrt{1-\alpha}+{\sqrt{2 \theta_{1}}}^{2}}{9} \leq \frac{\theta_{1} 1-\alpha}{2 \theta_{1}-1-\alpha}$, the upward IC will always be slack for cost structures investigated in this study $\left(\theta_{2} \geq \frac{\theta_{1} 1-\alpha}{2 \theta_{1}-1-\alpha}\right.$ as specified in Assumption 2); 
$\frac{2 \sqrt{1-\alpha}+{\sqrt{2 \theta_{1}}}^{2}}{9}>\frac{\theta_{1} 1-\alpha}{2 \theta_{1}-1-\alpha}$ gives the condition for $\theta_{1} \in\left(\frac{1}{2}, 1-\alpha\right)$ under which the upward IC will be violated; it can be simplified to $\theta_{1}>z$ where $z$ is the solution in $\left(\frac{1}{2}, 1-\alpha\right)$ to

$64 z^{4}-2721-\alpha z^{3}+1771-\alpha^{2} z^{2}+41-\alpha^{3} z+41-\alpha^{4}$.

The partial derivative of the profit function w.r.t. $f$ is

$-\frac{1}{2} f-c+\alpha \bar{r}-f+q_{1}+q_{2} \quad+\frac{\bar{r}-f+q_{1}}{2}+\frac{1}{2} f-c+\alpha \bar{r}-f+q_{1}+q_{2} \frac{\partial q_{1}}{\partial f} ;$ which is concave in $f$ when $q_{2}=0$. Substituting $q_{2}=0$ into the above formula and solving the corresponding first order condition yield

$f=\frac{\frac{c+\bar{r}}{2 \bar{r}}-\frac{\alpha \theta_{1}}{2 \theta_{1}-\sqrt{21-\alpha \theta_{1}}}}{1-\frac{\alpha \theta_{1}}{2 \theta_{1}-\sqrt{21-\alpha \theta_{1}}}} \bar{r} \frac{c+\bar{r}}{2 \bar{r}}$. Therefore $f^{*}=\max \left\{c, \frac{\frac{c+\bar{r}}{2 \bar{r}}-\frac{\alpha \theta_{1}}{2 \theta_{1}-\sqrt{21-\alpha \theta_{1}}}}{1-\frac{\alpha \theta_{1}}{2 \theta_{1}-\sqrt{21-\alpha \theta_{1}}}} \bar{r}\right\}$.

When the platform price is an interior solution, i.e., $f^{*}=\frac{\frac{c+\bar{r}}{2 \bar{r}}-\frac{\alpha \theta_{1}}{2 \theta_{1}-\sqrt{21-\alpha \theta_{1}}} \bar{r}}{1-\frac{\alpha \theta_{1}}{2 \theta_{1}-\sqrt{21-\alpha \theta_{1}}}}$,

$q_{1}=\frac{\sqrt{21-\alpha \theta_{1}}+1-\alpha 2+\sqrt{21-\alpha \theta_{1}}}{2 \alpha-2^{2} \theta_{1}-41-\alpha} \bar{r}-c$ and $q_{2}=0$. And, thus the profit is

$\frac{\bar{r}-c^{2} \theta_{1}}{42-\alpha \theta_{1}-4 \sqrt{21-\alpha \theta_{1}}}$ 
When the price for the platform product is a corner solution, i.e. $f^{*}=c, q_{1}=\frac{\sqrt{21-\alpha \theta_{1}}+1-\alpha}{2 \theta_{1}-1-\alpha} \bar{r}-c$ and $q_{2}=0$. Thus the profit is $\frac{\alpha}{4}\left[\frac{\bar{r}-c \sqrt{21-\alpha \theta_{1}}+2 \theta_{1}}{2 \theta_{1}-1-\alpha}\right]^{2}$.

\section{Proof of Proposition 2:}

Note that the RHS of the downward IC condition $\left(\frac{\bar{r}-f+2 q_{1}}{3}\right)^{2} 1-\alpha-\frac{1}{2} \theta_{2} q_{1}^{2}$ is the IR condition for the high-cost developer when the platform firm induces both developers to contribute equally to the demand for its product. Violation of the upward IC condition, i.e., $\left(\frac{\bar{r}-f+2 q_{1}}{3}\right)^{2} 1-\alpha-\frac{1}{2} \theta_{2} q_{1}^{2}>0$ with $q_{1}=\frac{1-\alpha+\sqrt{21-\alpha \theta_{1}}}{2 \theta_{1}-1-\alpha} \bar{r}-f \quad$ implies that the solution to $\left(\frac{\bar{r}-f+2 q_{1}}{3}\right)^{2} 1-\alpha-\frac{1}{2} \theta_{2} q_{1}^{2}=0$ is larger $\left(q_{1}=\frac{41-\alpha+3 \sqrt{21-\alpha \theta_{2}}}{9 \theta_{2}-81-\alpha} \bar{r}-f\right)$ than $\left(\frac{\bar{r}-f+q_{1}}{2}\right)^{2} 1-\alpha-\frac{1}{2} \theta_{1} q_{1}^{2}=0$ $\left(q_{1}=\frac{1-\alpha+\sqrt{21-\alpha \theta_{1}}}{2 \theta_{1}-1-\alpha} \bar{r}-f\right)$ for a given $f$. This implies that when the upward IC condition binds for $q_{1}=\frac{1-\alpha+\sqrt{21-\alpha \theta_{1}}}{2 \theta_{1}-1-\alpha} \bar{r}-f$ and $q_{2}=0$, the platform firm would induce a larger overall quality $q_{1}+q_{2}$ as well as a higher profit (comparing $\left(\frac{\bar{r}-f+q_{1}+q_{2}}{3}\right)\left(f-c+2 \alpha\left(\frac{\bar{r}-f+q_{1}+q_{2}}{3}\right)\right)$ and $\left(\frac{\bar{r}-f+q_{1}}{2}\right)\left(f-c+\alpha\left(\frac{\bar{r}-f+q_{1}}{2}+q_{2}\right)\right)$ for a given $\left.f\right)$ had it instead induced both developers to be the demand contributors with producing at the same quality. 
Lemma 2 indicates that the separating strategy is implementable and would generate a higher profit than the pooling strategy for $\theta_{1}<\frac{2}{3} 1-\alpha$ and $\theta_{2}<21-\alpha$. Under the same cost structures, shutting

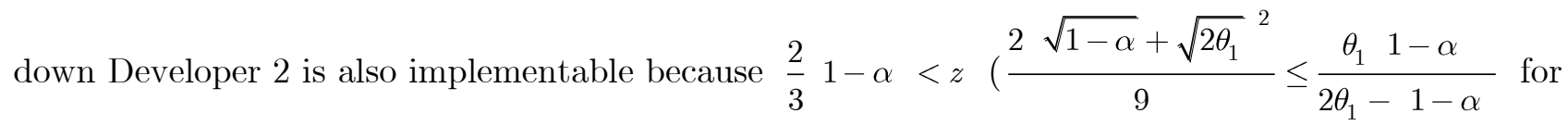
$\left.\theta_{1}<\frac{2}{3} 1-\alpha\right)$

Therefore, for a given $f$, the platform firm's profit is given by $\left(\frac{\bar{r}-f+q_{1}+q_{2}}{3}\right)\left(f-c+2 \alpha\left(\frac{\bar{r}-f+q_{1}+q_{2}}{3}\right)\right)$ under the separating strategy; or $\left(\frac{\bar{r}-f+q_{1}}{2}\right)\left(f-c+\alpha \frac{\bar{r}-f+q_{1}}{2}\right)$ if the inefficient developer is shut down.

Denote $x=\frac{\bar{r}-f+q_{1}+q_{2}}{3}$ and $y=\frac{\bar{r}-f+q_{1}}{2}$, and substituting $q_{1}$ and $q_{2}$ in (3.8) and $q_{1}=\frac{1-\alpha+\sqrt{21-\alpha \theta_{1}}}{2 \theta_{1}-1-\alpha} \bar{r}-f$ into $x$ and $y$ respectively yield $2 x=\frac{2 \alpha 2-2 \alpha+9 \theta_{1} \sqrt{21-\alpha \theta_{2}}}{27 \theta_{1} \sqrt{21-\alpha \theta_{2}}-21-\alpha \quad 4-4 \alpha+3 \sqrt{21-\alpha \theta_{2}}}$ and $y=\frac{2 \theta_{1}+\sqrt{21-\alpha \theta_{1}}}{22 \theta_{1}-1+\alpha}$.

It can be verified that $2 x<y$ when $\theta_{1}<\frac{2}{3} 1-\alpha$ and $\theta_{2}<21-\alpha$, implying that shutting down the inefficient developer can generate higher profit.

\section{Proof of Proposition 3:}

Under the quality delegation region, since $q_{1}=\frac{\bar{r}-f 1-\alpha}{2 \theta_{1}-1-\alpha}$, and $q_{2}=\frac{\bar{r}-f 1-\alpha}{2 \theta_{1}-1-\alpha} \frac{\theta_{1}}{\theta_{2}}$, the platform firm's profit is given by $\frac{\theta_{1}}{2 \theta_{1}-1-\alpha}\left(f-c+\alpha \frac{\theta_{1}\left(1+\frac{1-\alpha}{\theta_{2}}\right)}{2 \theta_{1}-1-\alpha} \bar{r}-f\right) \bar{r}-f$. 
Under the quality control region, for the case of $q_{1}=\frac{1-\alpha+\sqrt{21-\alpha \theta_{1}}}{2 \theta_{1}-1-\alpha} \bar{r}-f$ and $q_{2}=0$, the platform firm's profit is given by $\frac{\theta_{1}+\sqrt{\frac{1}{2} 1-\alpha \theta_{1}}}{2 \theta_{1}-1-\alpha}\left(f-c+\alpha \frac{\theta_{1}+\sqrt{\frac{1}{2} 1-\alpha \theta_{1}}}{2 \theta_{1}-1-\alpha} \bar{r}-f\right) \bar{r}-f$. For a given $\bar{r}>f \geq c$, the difference in the profits under the two regimes are given by $\left.\frac{\theta_{1}+\frac{\sqrt{21-\alpha \theta_{1}}}{2 \theta_{1}-1-\alpha}\left(f-c+\alpha \frac{\theta_{1}+\frac{\sqrt{21-\alpha \theta_{1}}}{2 \theta_{1}-1-\alpha}}{r}-f\right) \bar{r}-f-}{\theta_{1}\left(1+\frac{1-\alpha}{\theta_{2}}\right)} \bar{r}-f\right) \bar{r}-f$
$\frac{\theta_{1}}{2 \theta_{1}-1-\alpha}\left(f-c+\alpha \frac{\left(-\alpha \theta_{1}-1-\alpha\right.}{2}\right.$
$=\frac{\left.\sqrt{\frac{1}{2} 1-\alpha \theta_{1}} f-c+\left[\frac{1-\alpha}{2}+\sqrt{21-\alpha \theta_{1}}-\frac{1-\alpha \theta_{1}}{\theta_{2}}\right] \frac{\alpha \theta_{1} \bar{r}-f}{\left(\theta_{1}-(-\alpha)\right.}\right)_{(-f}}{2 \theta_{1}-(-\alpha)}$,

which is positive because $\frac{1-\alpha}{2}+\sqrt{21-\alpha \theta_{1}}-\frac{1-\alpha \theta_{1}}{\theta_{2}}>0$ by Assumption 2 . 